\title{
Residue reduction and intersurface interaction on single graphene sheets
}

Linda Karlsson, Jens Birch, Aurelija Mockuté, Arni Sigurdur Ingason, Huy Q. Ta, Mark H.

Rummeli, Johanna Rosén and Per O. Å. Persson

\section{Linköping University Post Print}

\section{Tweet}

N.B.: When citing this work, cite the original article.

Original Publication:

Linda Karlsson, Jens Birch, Aurelija Mockuté, Arni Sigurdur Ingason, Huy Q. Ta, Mark H. Rummeli, Johanna Rosén and Per O. Å. Persson, Residue reduction and intersurface interaction on single graphene sheets, 2016, Carbon, (100), 345-350.

http://dx.doi.org/10.1016/j.carbon.2016.01.007

Copyright: Elsevier

http://www.elsevier.com/

Postprint available at: Linköping University Electronic Press

http://urn.kb.se/resolve?urn=urn:nbn:se:liu:diva-126123

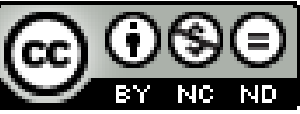




\title{
Residue reduction and intersurface interaction on single graphene sheets
}

Linda H. Karlsson ${ }^{1}$, Jens Birch ${ }^{1}$, Aurelija Mockute ${ }^{1}$, Arni S. Ingason ${ }^{1}$, Huy Q. Ta ${ }^{2,3}$, Mark H. Rummeli $^{4,5,6}$, Johanna Rosen ${ }^{1}$ and Per O.A.. Persson ${ }^{1}$

${ }^{1}$ Department of Physics, Chemistry and Biology, Linköping University, SE-581 83 Linköping, Sweden,

${ }^{2}$ IBS Center for Integrated Nanostructure Physics, Institute for Basic Science, Sungkyunkwan University, Suwon 440-746, Korea

${ }^{3}$ Department of Energy Science, Department of Physics, Sungkyunkwan University, Suwon 440-746, Korea

${ }^{4}$ College of Physics, Optoelectronics and Energy \& Collaborative Innovation Center of Suzhou Nano Science and Technology, Soochow University, Suzhou 215006, China

${ }^{5}$ IFW Dresden, Institute for Complex Materials, P.O. Box D-01171 Dresden, Germany

${ }^{6}$ Centre of Polymer and Carbon Materials, Polish Academy of Sciences, M. CurieSklodowskiej 34, Zabrze 41-819, Poland

\begin{abstract}
Large regions of pristine graphene are essential to applications which rely on the ideal graphene properties. Common methods for transferring chemical vapour deposition grown graphene to suitable substrates leaves metal oxide particles and poly(methyl methacrylate) (PMMA) residues on opposing surfaces, which degrade the properties. A common method to reduce the residues include annealing in vacuum or in argon, however, residues remain on the graphene
\end{abstract}

*Corresponding author. Tel: 46 (0)13 28 5772. E-mail: likar@ifm.liu.se (Linda H. Karlsson) 
sheet. The present investigation reports on the metal oxide particle ripening and PMMA decomposition on a single graphene sheet during in-situ annealing up to $1300{ }^{\circ} \mathrm{C}$ in a transmission electron microscope. It is shown that the PMMA residues are increasingly reduced at elevated temperatures although the reduction is strongly correlated to the metal oxide particle coverage on the opposing graphene surface. This is shown to occur as a consequence of an electrostatic interaction between the residues and that this prevents the establishment of large clean areas.

\section{Introduction}

Graphene is the first discovered stable two dimensional material [1] and is intensely studied due to its unique properties. A common method for manufacturing the graphene is through chemical vapour deposition (CVD) onto a $\mathrm{Cu}$ substrate with subsequent transfer to other, technically more relevant, substrates by applying a poly(methyl methacrylate) (PMMA) support to the graphene surface. The $\mathrm{Cu}$ substrate is then removed from the graphene by chemical etching using e.g. $\mathrm{FeCl}_{3}[2,3,4], \mathrm{HNO}_{3}[4,5], \mathrm{Fe}\left(\mathrm{NO}_{3}\right)_{3}[2,3,4]$ or $\left(\mathrm{NH}_{4}\right)_{2} \mathrm{SO}_{8}[2,4]$ while the PMMA support is reduced after the transfer process using acetone [2, 4, 5, 6, 7, 8]. These process steps leave a varying amount of residues, in the form of e.g. hydrocarbons and carboxyl groups from the PMMA support [9] and $\mathrm{FeO}_{\mathrm{x}}$ metal oxide particles from the acid if $\mathrm{FeCl}_{3}$ or $\mathrm{Fe}\left(\mathrm{NO}_{3}\right)_{3}$ is used $[6,7,10]$. The residues associated with the transfer process are known to affect the graphene's properties [2, 3, 4, 5, 6, 7, 11, 12]. PMMA residues effectively introduce p-type doping to the graphene sheet [13], decrease carrier mobility and cause wrinkles, tears and cracks [3, 4, 11]. Additionally, the metal oxide particles limit the thermal conductivity and also cause p-type doping [10]. Together, residues remaining on the surfaces after processing limit the ultimate properties of the graphene. Removal of the residues is thus imperative and several different methods have been reported. The most common method to reduce the amount of PMMA residues from the graphene sheet is through annealing in vacuum or in an $\mathrm{Ar} / \mathrm{H}_{2}$ atmosphere [2, 3, 4, 5, 6, 7, 8] as PMMA decomposes during annealing. Annealing graphene with PMMA residues at $160{ }^{\circ} \mathrm{C}$ causes a first decomposition of PMMA, while at $300^{\circ} \mathrm{C}$ random scissions in the polymer chain occur [9, 14]. However, PMMA residues are not completely removed after annealing at $300{ }^{\circ} \mathrm{C}[15,16]$ and have been shown to bind covalently to defects 
in the graphene lattice which may inhibit the decomposition $[9,16]$. Annealing at $500{ }^{\circ} \mathrm{C}[17$, 18] or $700{ }^{\circ} \mathrm{C}$ [19] increases the area of clean graphene regions and removes most of the PMMA residues. This is influenced by the healing of graphene at temperatures above $500{ }^{\circ} \mathrm{C}$ which is reported to remove the defect sites where the PMMA residues may be attached [20, 21].

During annealing of graphene with PMMA residues and metal oxide particles at high temperatures, the metal oxide particles may interfere with the decomposition of PMMA residues. This has been observed as metal atoms deposited on graphene sheets attach to the PMMA residues [19, 22, 23], which indicates a preferential interaction between the metal atoms and the PMMA residues. Annealing at $700{ }^{\circ} \mathrm{C}$ [19] or $900{ }^{\circ} \mathrm{C}$ [23] removes the PMMA residues and results in agglomeration of the metal atoms to particles. In general, the interaction between the particles and PMMA residues is discussed with respect to in-plane interaction, but not outof-plane through the graphene sheet. However, any interaction which might occur between the PMMA residues and the particles through the graphene sheet has not been examined.

In this report we investigate suspended Cu CVD grown single graphene sheets by in-situ annealing up to $1300{ }^{\circ} \mathrm{C}$ in a transmission electron microscope (TEM). In particular, we correlate the decomposition of PMMA residues at high temperatures to the metal oxide particle morphology on the opposite surface of the graphene sheets. As the PMMA residues remain on one side of the graphene sheet, the amount and area of metal oxide particles on the opposing graphene surface is tuned by means of sub-monolayer sputter deposition. Through this, it is concluded that there is a strong correlation between PMMA residues decomposition efficiency at elevated temperatures and metal oxide particle morphology. It is shown that this occurs as a consequence of electronic exchange through the graphene sheet and identifies improved metal oxide particle removal as means to significantly increase the regions of pristine graphene.

\section{Experimental procedure}

\subsection{Sample preparation}

Graphene was grown on polished copper using atmospheric pressure CVD with methane (99.999 \%) as the feedstock. Prior to growth, a $100 \mu \mathrm{m}$ thick copper foil (from Nilaco, 99.96 \%) was annealed at $1060{ }^{\circ} \mathrm{C}$ with $1000 \mathrm{sccm}$ Ar and $200 \mathrm{sccm} \mathrm{H}_{2}$ for $2 \mathrm{~h}$, then polished using 
a chemical mechanical polishing route. The polished copper was then mounted in a 2 inch quartz tube reactor and heated up to $1060{ }^{\circ} \mathrm{C}$ for $40 \mathrm{~min}$ with constant flows of $1000 \mathrm{sccm} \mathrm{Ar}$ and $200 \mathrm{sccm} \mathrm{H}_{2}$. After reaching $1060{ }^{\circ} \mathrm{C}$, the sample was annealed for $1 \mathrm{~h}$ without changing the gas flow. During the atmospheric pressure CVD growth, the $\mathrm{H}_{2}$ gas was reduced to $50 \mathrm{sccm}$ and $3 \mathrm{sccm}$ of $\mathrm{CH}_{4}$ (1 \% diluted in $\mathrm{Ar}$ ) was injected. After growth, the $\mathrm{CH}_{4}$ was turned off and the chamber was cooled to room temperature. This method produces large single layer graphene sheets [24].

The graphene sheets were then transferred to DENSsolutions single tilt heater chips. In order to protect the graphene film during Cu etching, a PMMA solution (950 k C4,) was spin-coated on the graphene/ $\mathrm{Cu}$ at $1000 \mathrm{rpm}$ for $60 \mathrm{~s}$. To etch the $\mathrm{Cu}$ foil, the sample was floated in copper etchant (CE-100, Transene) for about $30 \mathrm{~min}$. After thoroughly rinsing in deionized water, the PMMA/graphene layer was fished onto TEM heater chip. It was then dried in an oven for 5 minutes at $120{ }^{\circ} \mathrm{C}$. Finally, the PMMA was removed by exposure to acetone. The success of each transfer was then confirmed by SEM imaging.

\subsection{DC magnetron sputtering of $\mathrm{Cr}$}

Sub-monolayer Cr was deposited on the PMMA free side of the graphene sheets by dc magnetron sputtering from a 3 inch Cr (99.995 \% purity) target at room temperature, with 16.4 sccm of Ar introduced up to a partial pressure of 4.7 Pa. A high partial pressure was chosen to limit the kinetic energy of the $\mathrm{Cr}$ atoms during deposition. The base pressure of the system was under $7 \mu \mathrm{Pa}$. The depositions were carried out in a power controlled mode at $6 \mathrm{~W}$ at substratetarget distance of $\sim 15 \mathrm{~cm}$ for 1, 4, and $8 \mathrm{~min}$. The deposition times were selected based on growth rate calibration on Si substrates and correspond to $0.1,0.4$, and 0.8 monolayers of $\mathrm{Cr}$, respectively. In order to reach stable sputtering conditions, the $\mathrm{Cr}$ target was presputtered with source shutters closed for $\sim 5$ min prior each deposition with heater chip faced away from the flux, ensuring that absolutely no Cr was deposited during presputtering.

\subsection{In-situ Annealing}

The chips were analysed with the Linköping double corrected and monochromated FEI Titan ${ }^{3}$ 60-300 operated at $60 \mathrm{kV}$ in both scanning transmission electron microscopy (STEM) and 
monochromated TEM modes. Low-voltage $(60 \mathrm{kV})$ was applied for imaging and spectroscopy as it reduces knock-on damage [25]. Electron energy-loss spectroscopy (EELS) was performed using a GIF Quantum ERS spectrometer in dual-EELS mode.

An as-prepared, non-deposited sample was heated to $300^{\circ} \mathrm{C}$ employing a DENSsolutions single tilt holder [26], and left for one hour before lowering the temperature to ambient temperature where TEM imaging was performed. The sample was then annealed at $400{ }^{\circ} \mathrm{C}$ for one hour before lowering to room temperature and performing imaging. This was repeated in steps of 50 ${ }^{\circ} \mathrm{C}$ up to $1000{ }^{\circ} \mathrm{C}$, after which the sample was heated to $1100{ }^{\circ} \mathrm{C}$ and $1200{ }^{\circ} \mathrm{C}$ before imaging. For each temperature, a new region was imaged so any effect of the electron beam was minimized. Prior to and after annealing, the sample was investigated by monochromated TEM and EELS.

The Cr-deposited samples were subsequently investigated by STEM high-angle annular dark field (HAADF) imaging, after which the samples were annealed at $500{ }^{\circ} \mathrm{C}$ for 16 minutes. The temperature was then increased to $1300{ }^{\circ} \mathrm{C}$ in steps of $50{ }^{\circ} \mathrm{C}$ and for 16 minutes at each temperature. STEM HAADF images were acquired for each temperature after 1, 2, 4, 8, and 16 minutes. The region investigated was changed three times to limit any effect of the electron beam on the sample. Before and after heating, EELS spectrum imaging (SI) was performed to investigate the changes in microstructure and chemistry of the graphene and PMMA residues.

\subsection{Data analysis}

Analysis of the EELS data was performed using Digital Micrograph (DM). Calculation of the chemical shifts in the C-K edge in an EELS spectrum image (SI) was performed by NLLSfitting of the peaks in the core-loss SI. 
The area of the clean regions was determined by using Image ${ }^{1}$ with a minimum area of $1 \mathrm{~nm}^{2}$. The monochromated TEM images were filtered by applying a FFT mask to remove contrast variations from the monochromated beam prior to analysis.

\section{Results and discussion}

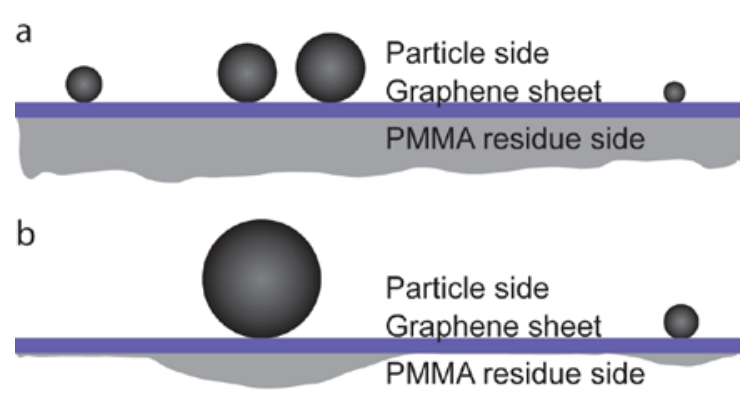

Figure 1. Schematic of graphene with PMMA residues and metal oxide particles on either side of the graphene sheet, a) prior to annealing and b) after annealing.

Initially, the as-prepared single layer graphene sheets are almost completely covered by PMMA residues with randomly distributed metal oxide particles. The metal oxide particles are attached to one surface (metal oxide particle side) of the sheet, while the PMMA residues are attached to the other (PMMA residues side), see schematic cross-section in Figure 1a. The as-prepared sample is visualized in projection by monochromated TEM, see Figure 2a.

\footnotetext{
${ }^{1}$ W. Rasband, ImageJ, U.S. National Institutes of Health, Bethesda, Maryland, USA, 19972014. URL http://imagej.nih.gov/ij/
} 


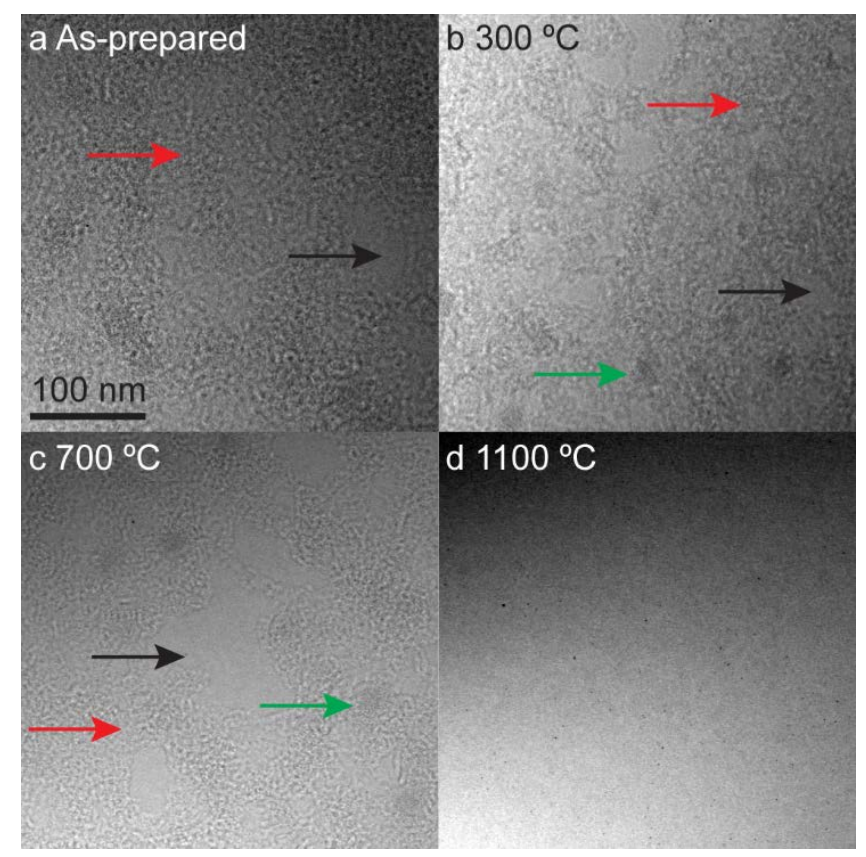

Figure 2. TEM images of different regions of a graphene sheet with PMMA residues and metal oxide particles prior to annealing (a) and during annealing at $300{ }^{\circ} \mathrm{C}(\mathrm{b}), 700{ }^{\circ} \mathrm{C}(\mathrm{c})$, and 1100 ${ }^{\circ} \mathrm{C}(d)$. The black arrows indicate regions of clean graphene, red arrows PMMA residues, and green arrow metal oxide particles.

In Figure 2, the overall contrast is nonuniform due to the monochromated electron beam which is dispersed diagonally across the images. The metal oxide particles are identified as dark spots while the PMMA residues are the larger, disordered and unevenly distributed regions, where the contrast is varying as a consequence of thickness variations of the PMMA residues. Regions with homogenous contrast constitute areas of clean graphene. No regions with multiple graphene layers were observed. Prior to annealing, the average area of such regions is on the order of tens of $\mathrm{nm}^{2}$.

Figures 2b-d illustrate typical changes occurring in the as-prepared sample and the gradual appearance of clean graphene regions, as the PMMA residues are reduced during thermal annealing from $300{ }^{\circ} \mathrm{C}$ (Figure 2b) to $1100{ }^{\circ} \mathrm{C}$ (Figure 2d). Annealing at $300{ }^{\circ} \mathrm{C}$ causes no obvious change to the PMMA residues (compare Figures 2a and b). Thus the common method of annealing at $300{ }^{\circ} \mathrm{C}$ in vacuum is an inadequate process to remove the PMMA residues. The area of the clean graphene regions increases slightly when raising the temperature to $700{ }^{\circ} \mathrm{C}$ (Figure 2c) indicating that the PMMA residues are reduced. Additionally, there is a reduction 
in number and increase in average area of the metal oxide particles, which indicates a ripening process on the metal oxide particle side, see Figures $2 \mathrm{~b}-\mathrm{d}$. The reduction in residue coverage on the graphene sheet is however not uniform. The outlines of some clean graphene regions remains intact while other regions expand, increasing the overall area distribution. When the temperature is increased to $1100{ }^{\circ} \mathrm{C}$ a significant reduction of the residues occurs and the remaining residues are organized in a web-like appearance on the graphene sheet. Consequently, an increase in regions of clean graphene is observed, see Figure $2 \mathrm{~d}$, where the areas are approximately $650 \mathrm{~nm}^{2}$ at $1100{ }^{\circ} \mathrm{C}$.

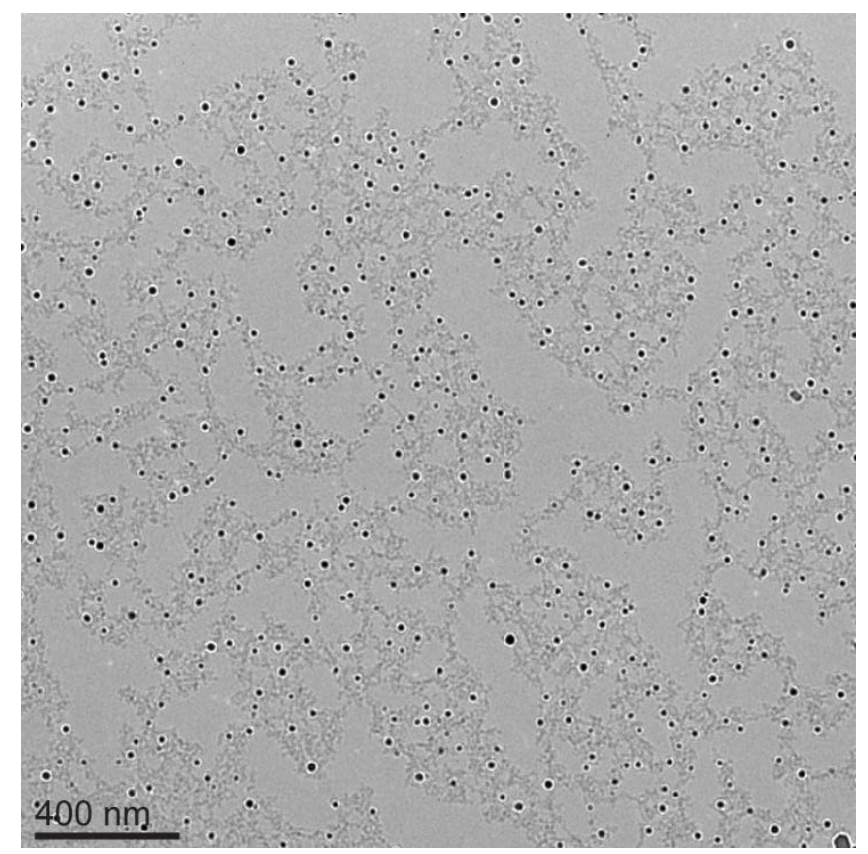

Figure 3. TEM image of graphene showing PMMA residues and metal oxide particles after annealing to $1200{ }^{\circ} \mathrm{C}$. For contrast purposes, a strong defocus was applied, causing Fresnel fringes around the metal oxide particles.

At $1200{ }^{\circ} \mathrm{C}$ the area of the clean graphene regions increases to an order of $10^{4} \mathrm{~nm}^{2}$, as seen in Figure 3 which shows a representative overview of the as-prepared sample. In Figure 3, the web-like appearance of the PMMA residues is apparent, and the distribution of PMMA residues and metal oxide particles is entirely random and assumes no relation to the graphene lattice. However, it is also observed that, although attached to opposite sides of the graphene sheet, there is a correlation between metal oxide particles and PMMA residues such that they exclusively overlap in projection. This indicates an interaction between the PMMA residues and metal oxide particles through the graphene sheet. 


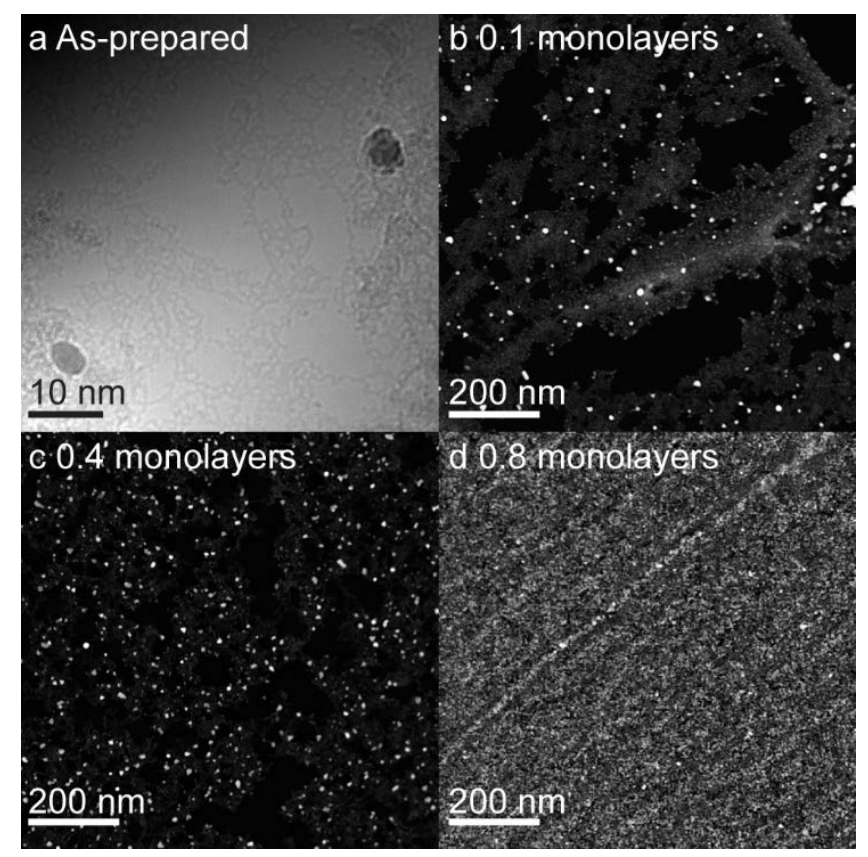

Figure 4. TEM (a) and STEM HAADF (b-d) images of graphene with PMMA residues deposited with 0 (a), 0.1 (b), 0.4 (c), and 0.8 (d) monolayers of $\mathrm{Cr}$ and annealed at $1200^{\circ} \mathrm{C}$.

Adding varying thicknesses of sub-monolayer of $\mathrm{Cr}$ to the metal oxide particle side affects the resulting structure obtained after annealing to $1200{ }^{\circ} \mathrm{C}$, see Figure 4 . The annealed, as-prepared sample is shown in Figure 4a as imaged by monochromated TEM, while the remaining three images show the appearance of the deposited and annealed samples using STEM HAADF. For 0.1 monolayers (Figure 4b) and 0.4 monolayers (Figure 4c), the deposited $\mathrm{Cr}$ is found to ripen into metal oxide particles, where the number of metal oxide particles is higher for 0.4 monolayers as a consequence of the additional deposition. However, the extended deposition of 0.8 monolayers (Figure 4d) appears to form a coating on the sample and that ripening is inhibited. Note that this is the result after annealing at $1200^{\circ} \mathrm{C}$. Also noteworthy in this context is the apparent banded organization of the deposited material. Since Cr exhibits strong mass contrast in STEM HAADF images, it is inferred that the present contrast variations originate from an inhomogeneous $\mathrm{Cr}$ distribution rather than PMMA residues thickness variations. The diagonal lines in Figure 4d indicate that the deposited Cr metal oxide particles are aligned on the metal oxide particle side to mirror the PMMA residues on the opposing side. PMMA residues typically exhibit a wavy topography originating from the transfer process. 
EELS analysis of the annealed, deposited samples confirmed the presence of $\mathrm{C}, \mathrm{O}, \mathrm{Fe}, \mathrm{Cr}$ as well as traces of $\mathrm{Ca}$, see Table 1 .

Table 1. Relative composition of graphene deposited with 0.1, 0.4, and 0.8 monolayers of $\mathrm{Cr}$ after annealing to $1300^{\circ} \mathrm{C}$.

\begin{tabular}{llll}
\hline \multicolumn{4}{c}{ Composition (Rel. \\
%) } \\
\hline Element & 0.1 monol. & 0.4 monol. & 0.8 monol. \\
\hline $\mathrm{Cr}$ & $1.0 \pm 0.1$ & $7.6 \pm 0.9$ & $12.4 \pm 1.5$ \\
$\mathrm{C}$ & $78.5 \pm 10.1$ & $71.9 \pm 8.9$ & $65.2 \pm 7.9$ \\
$\mathrm{O}$ & $14.1 \pm 1.8$ & $13.4 \pm 1.7$ & $18.0 \pm 2.2$ \\
$\mathrm{Fe}$ & $2.1 \pm 0.3$ & $6.2 \pm 0.8$ & $4.2 \pm 0.5$ \\
$\mathrm{Ca}$ & $4.3 \pm 0.6$ & $0.9 \pm 0.1$ & $0.2 \pm 0.0$ \\
\hline
\end{tabular}

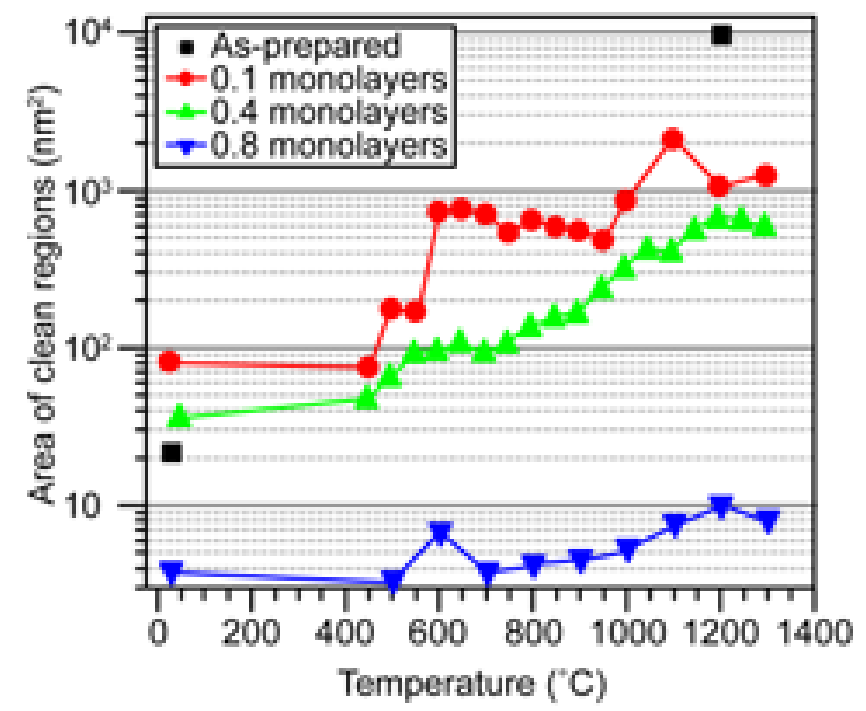

Figure 5. Average area of clean regions on the graphene sheet as a function of temperature and $\mathrm{Cr}$ coverage (note the logarithmic scale).

The average area of the clean graphene regions is quantitatively correlated to temperature and deposition thickness, see Figure 5. Here it is apparent that the area of the clean graphene region depends strongly on the metal oxide particle coverage throughout the annealing experiment. The variations of PMMA residues coverage prior to annealing are due to local thickness 
variations and to different coverages of the metal oxide particles. At 0.8 monolayers of Cr, the metal oxide particles cover almost the entire surface and thus the area of the clean regions is limited. The average clean area of the sample with 0.8 monolayers is clearly lower than the samples with 0.1 monolayers and 0.4 monolayers of Cr, while the difference between these samples is smaller but noticeable. A sudden increase at $1100{ }^{\circ} \mathrm{C}$ for 0.1 monolayers is due to change of investigated region on the sample. For the as-prepared sample, there is little difference compared to the deposited samples prior to annealing, however, after annealing to $1200{ }^{\circ} \mathrm{C}$ the as-prepared sample exhibits a substantial increase in clean graphene regions. Independent of sample, the average clean region increases significantly at temperatures above $1000{ }^{\circ} \mathrm{C}$. This further supports that metal oxide particles on the adjacent graphene surface inhibit the reduction of PMMA residues upon heating.

Cleaning graphene by annealing to $1200{ }^{\circ} \mathrm{C}$ causes the PMMA residues to primarily dissociate from the graphene sheet, leaving large clean graphene regions. The PMMA residues reduction is decidedly dependent on the amount of metal oxide particles on the opposing surface. More metal oxide particles on the adjacent surface limit the PMMA residues desorption and it would therefore be beneficial to choose a preparation method which substantially reduces the number of metal oxide particles. Due to this correlation between metal oxide particle coverage and PMMA residues reduction, it appears that there is an interaction between the PMMA residues and the metal oxide particles through the graphene sheet. To pursue this, the chemistry in the vicinity of the metal oxide particles was investigated by STEM-EELS SI.

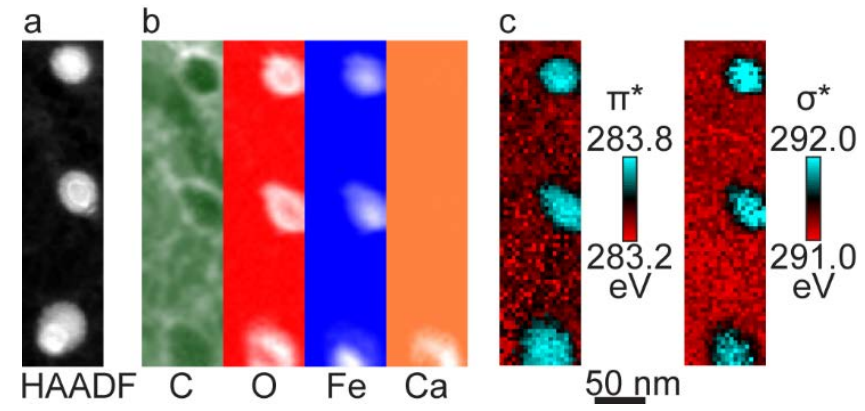

Figure 6. Elemental and chemical maps of the annealed, as-prepared sample a) STEM HAADF image of three metal oxide particles with elemental maps of $\mathrm{C}, \mathrm{O}, \mathrm{Fe}$, and $\mathrm{Ca}(\mathrm{b})$. The chemical shift of $C$-K $\pi^{*}$-peak and $\sigma^{*}$-peak are shown in (c). 
Figure 6 shows a STEM HAADF image of a region on the annealed, as-prepared sample exhibiting three metal oxide particles on the graphene sheet and PMMA residues (Figure 6a) with corresponding spectrum image extracted elemental maps. The particles are slightly elongated compared to the STEM HAADF image as a consequence of specimen drift during acquisition, see Figure 6b. Due to the substantial difference in thickness between the graphene sheet and the PMMA residues, almost all of the C-K signal is inferred to originate from the PMMA residues. Thus variations in the $\mathrm{C}-\mathrm{K}$ edge in the SI is interpreted as variations in the PMMA residues, see Figure 6b. A significant reduction of the C-signal intensity is revealed in the $\mathrm{C}$ elemental map beneath the metal oxide particles. This does not indicate a reduction in $\mathrm{C}$ but is an artefact as a significant portion of the electron beam is scattered by the metal oxide particles (and provides strong contrast in the STEM HAADF image) hence the local EELS intensity is reduced. A significant observation near the metal oxide particles is that $\mathrm{C}$ is present although the signal is reduced underneath the metal oxide particles, and it is inferred that the PMMA residues are equally thick underneath the centre and edges of the metal oxide particles. The increased $\mathrm{C}$ presence indicates that $\mathrm{C}$ is attracted by the metal oxide particles although attached to opposite sides of the graphene sheet, see schematic in Figure 1b. The interaction between PMMA residues and metal oxide particles is supported by the chemical shifts of the $\pi^{*}$ - and the $\sigma^{*}$-peaks of the C-K edge as recorded in Figure 6c. As with the C-elemental map, the apparent dark pixels in the $\sigma^{*}$-peak energy map at the metal oxide particles are artefacts, resulting from reduced signal to noise ratio and peak fitting challenges. The contrast at the metal oxide particle positions indicates a chemical shift of $0.11 \mathrm{eV}$ for the $\pi^{*}$-peak and $0.15 \mathrm{eV}$ for the $\sigma^{*}$-peak between the PMMA and the metal oxide particles.

The shift of the C-K edge is proposed to originate from an electrostatic interaction between metal oxide particles and PMMA residues through the graphene sheet. Charge exchange due to the interaction is suggested to cause a local shift of the Fermi level which can be observed as a chemical shift in the EEL spectra. This charge exchange serves to anchor the network of PMMA residues to the metal oxide particle sites. Hence a high temperature annealing process is more efficient in reducing PMMA residues in metal oxide particle free regions.

\section{Conclusions}


In this report the reduction and interaction of PMMA residues with metal oxide particles, positioned on opposing surfaces on the graphene sheet, have been investigated by TEM and STEM during annealing up to $1300{ }^{\circ} \mathrm{C}$. It is shown that metal oxide particles and PMMA residues interact electrostatically through the graphene sheet, inhibiting the decomposition of PMMA residues during the annealing process. Increasing the metal oxide particle coverage severely limits the PMMA residues decomposition, and hence this report shows that care must be taken to reduce the metal oxide particle concentration on graphene sheets. Modification of the transfer process by means of alternative substrate etchants has the potential to more efficiently remove metal oxide particles and PMMA residues and provide graphene sheets of improved quality.

\section{Acknowledgement}

The authors acknowledge the Swedish Research Council for funding under grants no. 6212012-4359, 622-2008-405 and 642-2013-8020, the Olle Engkvist foundation, as well as the Knut and Alice Wallenbergs Foundation for support of the electron microscopy laboratory in Linköping. The European Research Council under grant agreement no [258509] is also acknowledged. This work was supported by the IBS Korea. Birch, Rosen and Persson acknowledge the Swedish Government Strategic Research Area in Materials Science on Functional Materials at Linköping University (Faculty Grant SFO-Mat-LiU 2009-00971) for financial support.

Lars-Åke Näslund is acknowledged for his support in planning and performing depositions.

\section{References}

[1] K. S. Novoselov, A. K. Geim, S. V. Morozov, D. Jiang, Y. Zhang, S. V. Dubonos, et al., Electric field e_ect in atomically thin carbon films, Science 2004; 306(5696):666-669.

[2] J. Kang, D. Shin, S. Bae, H. H. Byung, Graphene transfer: key for applications, Nanoscale 2012; 4(18):5527-5537.

[3] A. Ambrosi, C. K. Chua, A. Bonanni, M. Pumera, Electrochemistry of graphene and related materials, Chem. Rev. 2014; 114(14):7150-7188. 
[4] C. Mattevi, H. Kim, M. Chhowalla, A review of chemical vapour depositionof graphene on copper, J. Mater. Chem. 2010; 21:3324-3334.

[5] A. Pirkle, J. Chan, A. Venugopal, D. Hinojos, C. W. Magnuson, S. McDonnell,et al., The effect of chemical residues on the physical and electrical properties of chemical vapor deposited graphene transferred to SiO2, Appl. Phys. Lett. 99 2011; 12:122108.

[6] S. Kataria, S. Wagner, J. Ruhkopf, A. Gahoi, H. Pandey, R. Bornemann, et al., Chemical vapor deposited graphene: From synthesis to applications, Phys. Status Solidi A 2014; 11:2439-2449.

[7] K. E. Whitener Jr, P. E. Sheehan, Graphene synthesis, Diamond Relat. Mater. 2014; 46:25-34.

[8] J.W. Suk, A. Kitt, C.W. Magnuson, Y. Hao, S. Ahmed, J. An, et al., Transfer of CVDgrown monolayer graphene onto arbitrary substrates, ACS Nano 2011; 5(9):6916-6924.

[9] Y.-C. Lin, C.-C. Lu, C.-H. Yeh, C. Jin, K. Suenaga, P.-W. Chiu, Graphene annealing: How clean can it be?, Nano Lett. 2012; 12(1):414-419.

[10] Z. T.Wu,W.W. Zhao,W. Y. Chen, J. Jiang, H. Y. Nan, X. T. Guo, et al., The influence of chemical solvents on the properties of cvd graphene, J. Raman Spectrosc. 2015; 46:21-24. [11] G. B. Barin, Y. Song, I. de F’atima Gimenez, A. G. S. Filho, L. S. Barreto, J. Kong, Optimized graphene transfer: Influence of polymethylmethacrylate (pmma) layer concentration and baking time on graphene final performance, Carbon 2015; 84(0):82-90. [12] K. S. Novoselov, V. I. Falko, L. Colombo, P. R. Gellert, M. G. Schwab, K. Kim, A roadmap for graphene, Nature 2012; 490:192-200.

[13] Y. Ahn, H. Kim, Y.-H. Kim, Y. Yi, S.-I. Kim, Procedure of removing polymer residues and its influences on electronic and structural characteristics of graphene, Appl. Phys. Lett. 2013; 102:091602.

[14] B. J. Holland, J. N. Hay, The effect of polymerisation conditions on the kinetics and mechanisms of thermal degradation of pmma, Polym. Degrad. Stabil. 2002; 77(3):435-439. [15] Z. Cheng, Q. Zhou, C. Wang, Q. Li, C. Wang, Y. Fang, Toward intrinsic graphene surfaces: A systematic study on thermal annealing and wet-chemical treatment of $\mathrm{SiO}_{2}$ supported graphene devices, Nano Lett. 2011; 11:767-771.

[16] W. Choi, Y.-S. Seo, J.-Y. Park, K. B. Kim, J. Jung, N. Lee, et al., Effect of annealing in $\mathrm{Ar} / \mathrm{H} 2$ environment on chemical vapor deposition-grown graphene transferred with poly (methyl methacrylate), IEEE Trans. Nanotechnol. 2015; 14(1):70-74.

[17] W. Xie, L.-T. Weng, K. M. Ng, C. K. Chan, C.-M. Chan, Clean graphene surface through high temperature annealing, 2015; Carbon 94:740-748. 
[18] R. Zan, C. Muryn, U. Bangert, P. Mattocks, P. Wincott, D. Vaughan, et al, Scanning tunnelling microscopy of suspended graphene, Nanoscale 2012; 4:3065-3068.

[19] L.-W. Huang, C.-K. Chang, F.-C. Chien, K.-H. Chen, P. Chen, F.-R. Chen, et al., Characterization of the cleaning process on a transferred graphene, J. Vac. Sci. Technol., A 2014; 32:050601.

[20] J. Chen, T. Shi, T. Cai, T. Xu, L. Sun, X. Wu, et al., Self healing of defected graphene, Appl. Phys. Lett. 2013; 102:103107.

[21] Z. H. Ni, H. M. Wang, Z. Q. Luo, Y. Y. Wang, T. Yu, Y. H. Wu, et al., The e_ect of vacuum annealing on graphene, J. Raman Spectrosc. 2009; 41:479-483.

[22] R. Zan, U. Bangert, Q. Ramasse, K. S. Novoselov, Interaction of metals with suspended graphene observed by transmission electron microscopy, J. Phys. Chem. Lett. 2012; 3:953958.

[23] R. Zan, U. Bangert, Q. Ramasse, K. S. Novoselov, Metal-graphene interaction studied via atomic resolution scanning transmission electron microscopy, Nano Lett. 2011; 11:10871092.

[24] V. L. Nguyen, B. G. Shin, D. L. Duong, S. T. Kim, D. Perello, Y. J. Lim, et al., Seamless Stitching of Graphene Domains on Polished Copper (111) Foil, Adv. Mater. 2015; 27:13761382.

[25] J. C. Meyer, F. Eder, S. Kurasch, V. Skakalova, J. Kotakoski, H. J. Park, et al., Accurate measurement of electron beam induced displacement cross sections for single-layer graphene, Phys. Rev. Lett. 2012; 108:196102.

[26] M. A. van Huis, N. P. Young, G. Pandraud, J. F. Creemer, D. Vanmaekelbergh, A. I. Kirkland, et al., Atomic Imaging of Phase Transitions and Morphology Transformations in Nanocrystals, Adv. Mater. 2009; 21:4992-4995. 